antiepileptic medications were ineffective; the ketogenic diet was unavailable in this center and had not been tried.

Of 314 children enrolled in the Far-East Asia Catastrophic Epilepsy (FACE) study group, age of onset of epilepsy was $<12$ months in 239 cases (80\%), epileptic spasms were the most frequent seizure type (in 42\%), followed by generalized tonic seizures (in 20\%) [1]. Epileptic syndromes included West syndrome (in 37\%), unclassified (21\%), Lennox-Gastaut (12\%), Dravet (4\%), and Rasmussen (2\%). Cortical dysplasia and chromosomal anomalies were the two most frequent causes of epilepsy, in $16 \%$ and $6 \%$, respectively; in almost one half of patients, the cause was unknown. Psychomotor development was retarded in $62 \%$ cases.

\title{
References.
}

1. Oguni H, et al. Brain Dev. 2013 Sep;35(8):786-92.

\section{PSYCHOGENIC NON-EPILEPTIC SEIZURES}

Investigators at the National Institute of Mental Health and Neurosciences, Bangalore, India, conducted a retrospective analysis of semiologic patterns of psychogenic non-epileptic seizures (PNES) diagnosed by video EEG in 56 children aged $<18$ years (mean age 12.3 yrs; range 2-17 yrs). Age at onset of PNES was 8.9 yrs (range 0.4-15.8 yrs); age at diagnosis 11.9 yrs (range 2-17 yrs); delay in diagnosis 3.2 yrs (range $0-15 \mathrm{yrs}$ ). Associated diagnoses included anxiety in $16 \%$, stress in $10 \%$, and depression $(10 \%)$. Coexistent epilepsy in $16 \%$ patients was complex partial in $8.9 \%$, generalized tonic-clonic in 5.4\%, and simple partial in 1.8\%. Prior to VEEG, $33(59 \%)$ patients were initially misdiagnosed as epilepsy and were treated with AEDs; in 14 patients $(25 \%)$ the initial diagnosis of PNES was unchanged after VEEG. EEG during a PNES showed various artifacts, depending on the type of movement or coma-like state. MRI performed in 14 patients with PNES alone was normal in $12(86 \%)$ and showed non-specific white matter signal changes or UBOs in 2. Characteristic signs of PNES were flexion/extension movements, moaning and gasping, tremors, flaccidity, vocalization, hyperventilation, and pelvic thrusting. Eyes were closed in $25(45 \%)$ and remained open during the PNES in $55 \%$. The EEG technician's simple motor commands were followed by $55 \%$ during the event. PNES was classified in 5 categories: I. Abnormal motor (hypermotor $(23 \%)$ and partial (14\%)); II. Affective/emotional behavior 3.6\% (moaning, grunting); III. Dialeptic 14\% (coma-like state, flaccidity); IV. Aura 5.4\% (subjective feeling, dizziness); V. Mixed (39\%). (Dhiman V, Sinha S, Rawat VS, et al. Children with psychogenic nonepileptic seizures (PNES): a detailed semiologic analysis and modified new classification. Brain Dev 2014 Apr;36(4):287-93).

COMMENTARY. Video-EEG is important in the diagnosis and differentiation of epileptic seizures from PNES. Epilepsy and PNES are coexistent in $16 \%$ of cases. In a previous semiologic analysis of 27 childhood PNES cases based on video-EEG monitoring, mean duration of PNES was longer compared to epileptic seizures, eyewitnesses were almost always present, eyes were closed at the onset in only $15 \%$ of events, tremor was the most frequent motor sign, and dialeptic PNES was most frequent among younger children [1]. 
References.

1. Szabo L, et al. Epilepsia. 2012 Mar;53(3):565-70.

\section{HUMAN METAPNEUMOVIRUS AND STATUS EPILEPTICUS}

Investigators at Cincinnati Children's Hospital, OH, report 2 toddlers, ages 15 and 18 months, with human metapneumovirus (hMPV) infection who presented in status epilepticus and later developed respiratory failure. Both patients recovered over 2 weeks with no sequelae. Infection with hMPV should be considered as a cause of seizures or encephalitis with respiratory symptoms in infants and children. (Webster DL, Gardner AH, Dye TJ, Chima RS. Status epilepticus: a possible association with human metapneumovirus infection. Pediatrics 2014 Mar;133(3):e747-50).

COMMENTARY. Neurological complications of human metapneumovirus infection are not mentioned in the 2012 edition of the AAP Redbook [1], and a review of seizures and hMPV in PubMed uncovers few reports. One earlier study reports an incidence of $6.3 \%$ of hMPV cases associated with seizures compared to $0.7 \%$ of patients infected with RSV ( $\mathrm{p}=0.031)$. hMPV may be associated with a spectrum of CNS disease ranging from febrile seizure to status epilepticus and severe, fatal encephalitis [2].

\section{References.}

1. AAP. Human Metapneumovirus. In: Pickering LK, et al, eds. Red Book: 2012 Report of the Committee on Infectious Diseases. 29th ed. Elk Grove Village, IL: AAP; 2012:509-10.

2. Arnold JC, et al. Pediatr Infect Dis J. 2009 Dec;28(12):1057-60.

\section{ENCEPHALITIDES}

\section{HERPES SIMPLEX AND NMDA ENCEPHALITIDES}

Investigators at University of Texas Southwestern Medical Center, Dallas, TX, report 2 male patients, an infant and adult, with confirmed herpes simplex encephalitis (HSE) and anti-NMDA receptor antibody encephalitis. Testing for anti-NMDA receptor antibodies and autoimmune disorder is recommended in patients with persistent encephalopathy, regression after initial improvement, or persistent movement disorders. Neuronal infections such as HSV may trigger subsequent anti-NMDA receptor antibody formation. Concomitant treatment or testing for immune-mediated encephalitis is indicated when treating viral encephalitis. (DeSena A, Graves D, Warnack W, Greenberg BM. Herpes simplex encephalitis as a potential cause of anti-N-methyl-D-aspartate receptor antibody encephalitis report of 2 cases. JAMA Neurology 2014;71(3):344-6).

COMMENTARY. The association of herpes simplex and anti-NMDA receptor antibody encephalitides is reported in 5 prospectively diagnosed patients ( 2 female) with relapsing post-herpes simplex encephalitis [1]. In 3 further retrospectively studied patients with HSE and NMDAR antibodies the frequency of autoantibodies increased over time, suggesting that HSE triggers NMDAR antibodies and brain autoimmunity [1]. 